\title{
A degenerate pseudo-parabolic equation with memory
}

\author{
Micol Amar ${ }^{1}$, Daniele Andreucci ${ }^{1 *}$, Roberto Gianni $^{2}$, Claudia Timofte $^{3}$ \\ ${ }^{1}$ Department of Basic and Advanced Sciences for Engineering, Sapienza - University of Roma, Via A. Scarpa 16, \\ 00161 Roma, Italy; \\ ${ }^{2}$ Department of Mathematics and Informatics \\ University of Firenze Via Santa Marta 3, 50139 Firenze, Italy; \\ ${ }^{3}$ University of Bucharest, Faculty of Physics. P.O. Box MG-11, Bucharest, Romania \\ ${ }^{*}$ Email address for correspondence: daniele.andreucci@sbai.uniroma1.it
}

Communicated by Nicola Bellomo

Received on 12 08, 2018. Accepted on 02 25, 2019.

\begin{abstract}
We prove the existence and uniqueness for a degenerate pseudo-parabolic problem with memory. This kind of problem arises in the study of the homogenization of some differential systems involving the Laplace-Beltrami operator and describes the effective behaviour of the electrical conduction in some composite materials.
\end{abstract}

Keywords: Existence and uniqueness, Pseudo-parabolic equations, Equations with memory.

AMS subject classification: 35K70, 35A01, 35B27

\section{Introduction}

Let $\Omega$ be an open bounded subset of $\mathbb{R}^{N}$ with regular boundary and let $T>0$. We study existence, uniqueness and regularity for the solution of the linear pseudo-parabolic problem with memory, given by

$$
\begin{array}{ll}
-\operatorname{div}\left(C \nabla u_{t}+A \nabla u+\int_{0}^{t} B(x, t-\tau) \nabla u \mathrm{~d} \tau\right)=f, & \text { in } \Omega \times(0, T) ; \\
u=0, & \text { on } \partial \Omega \times(0, T) ; \\
u(x, 0)=\bar{u}_{0}(x), & \text { in } \Omega,
\end{array}
$$

where $A, B, C: \Omega \times(0, T) \rightarrow \mathbb{R}^{N^{2}}$ are symmetric matrices, $A$ is positive definite and $C$ is only positive semi-definite. Here, $f: \Omega \times(0, T) \rightarrow \mathbb{R}$ and $\bar{u}_{0}: \Omega \rightarrow \mathbb{R}$ are given functions.

This problem, in the coercive case, arises in the study of electrical conduction in conductive media with microscopical dielectrical inclusions [1,2] and more recently in [3], in the case of the so-called connected/connected geometry; i.e., when the two conductive regions separated by the interface are both connected. The noncoercive case is expected to appear in the layered geometry; i.e., when the interfaces are layered (see [3, Remark 4.10]).

The coercive elliptic version of the previous problem arises as the homogenization limit in the study of some models for electrical conduction in biological tissues ( [4-7]) and has been considered from the point of view of the well-posedness in [8].

From the physical point of view, problem (1) describes the effective behaviour of the homogenized potential appearing in the macroscopic model of the composite material mentioned above. Here, the unknown $u$ represents the electrical potential and the driven electrical current

$$
-C(x, t) \nabla u_{t}-A(x, t) \nabla u-\int_{0}^{t} B(x, t-\tau) \nabla u(x, \tau) d \tau
$$

depends on the history of the electrical field $-\nabla u$, therefore it is non local in time. The term $-C(x, t) \nabla u_{t}$ originates from the displacement currents due to the presence of the dielectric interfaces. 
Diffusion problems with history are well known not only in the framework of biological applications but also in continuum mechanics; for instance, to model fluid flow and heat conduction (see, e.g., [9-18] and the references therein).

When problems of type (1) appear as homogenization limit, this automatically yields the existence of solutions. Here, we prove an independent result of existence and uniqueness under more general assumptions.

The paper is organized as follows. In Section 2, we state and prove the existence and uniqueness of the corresponding coercive problem. In Section 3, we prove the existence and uniqueness in the positive semi-definite case.

\section{The coercive case}

In this section, we will assume that $B \in L^{2}\left(0, T ; L^{\infty}\left(\Omega ; \mathbb{R}^{N^{2}}\right)\right)$ and $A, C \in L^{\infty}\left(\Omega \times(0, T) ; \mathbb{R}^{N^{2}}\right)$ are such that

$$
\begin{array}{ll}
\lambda|\xi|^{2} \leq A(x, t) \xi \cdot \xi \leq \Lambda|\xi|^{2}, & \text { for a.e. }(x, t) \in \Omega \times(0, T) \text { and } \forall \xi \in \mathbb{R}^{N} \\
\lambda|\xi|^{2} \leq C(x, t) \xi \cdot \xi \leq \Lambda|\xi|^{2}, & \text { for a.e. }(x, t) \in \Omega \times(0, T) \text { and } \forall \xi \in \mathbb{R}^{N},
\end{array}
$$

for suitable $0<\lambda<\Lambda<+\infty$. For the sake of brevity, we will employ the notation $\|v\|_{2}$ to denote the usual $L^{2}(\Omega \times(0, T))$-norm of a given function $v \in L^{2}(\Omega \times(0, T))$.

Theorem 2.1. Assume that $f \in L^{2}(\Omega \times(0, T))$ and $\bar{u}_{0} \in H_{0}^{1}(\Omega)$. Then, there exists a unique function $u \in L^{2}\left(0, T ; H_{0}^{1}(\Omega)\right)$ satisfying in the sense of distributions problem (1). The initial data is taken in the sense of $L^{2}(\Omega)$.

Proof. Consider the Banach space $X=L^{2}\left(0, T_{1} ; H_{0}^{1}(\Omega)\right)$, endowed with the usual norm

$$
\|u\|_{X}:=\left(\int_{0}^{T_{1}} \int_{\Omega}|\nabla u|^{2} d x d t\right)^{1 / 2}
$$

where $T_{1} \leq T$ will be chosen later. Let us introduce an operator $H$ acting on $X$ by means of $H(u)=\bar{w}$, where

$$
\bar{w}(x, t)=\bar{u}_{0}(x)+\int_{0}^{t} w(x, \tau) \mathrm{d} \tau, \quad \text { for a.e. }(x, t) \in \Omega \times\left(0, T_{1}\right),
$$

and $w$ is the weak solution of

$$
-\operatorname{div}(C(x, t) \nabla w)=\operatorname{div}\left(A(x, t) \nabla u(x, t)+\int_{0}^{t} B(x, t-\tau) \nabla u(x, \tau) \mathrm{d} \tau\right)+f(x, t),
$$

in $X$. Clearly, the operator $H$ is well defined; indeed, multiplying the previous equation by $w$ and integrating by parts, we obtain

$$
\begin{aligned}
& \lambda\|w\|_{X}^{2} \leq \int_{0}^{T_{1}} \int_{\Omega} C \nabla w \cdot \nabla w \mathrm{~d} x \mathrm{~d} t= \\
&-\int_{0}^{T_{1}} \int_{\Omega} A \nabla u \cdot \nabla w \mathrm{~d} x \mathrm{~d} t-\int_{0}^{T_{1}} \int_{\Omega}\left(\int_{0}^{t} B \nabla u \mathrm{~d} \tau\right) \cdot \nabla w \mathrm{~d} x \mathrm{~d} t+\int_{0}^{T_{1}} \int_{\Omega} f w \mathrm{~d} x \mathrm{~d} t \leq \\
& \frac{\Lambda}{2 \delta}\|u\|_{X}^{2}+\frac{\Lambda \delta}{2}\|w\|_{X}^{2}+\frac{T_{1}\|B\|_{L^{2}\left(0, T_{1} ; L^{\infty}(\Omega)\right)}^{2}\|u\|_{X}^{2}+\frac{\delta}{2}\|w\|_{X}^{2}+\frac{\|f\|_{2}^{2}}{2 \delta}+\frac{c \delta}{2}\|w\|_{X}^{2},}{}
\end{aligned}
$$


where $c$ is the Poincaré's constant. Therefore, taking $\delta=\frac{\lambda}{\Lambda+1+c}$, we can absorb the second, the fourth and the sixth term on the right-most hand side into the left-most hand side, thus obtaining

$$
\|w\|_{X}^{2} \leq \frac{\Lambda+1+c}{\lambda^{2}}\left[\left(\Lambda+T_{1}\|B\|_{L^{2}\left(0, T_{1} ; L^{\infty}(\Omega)\right)}^{2}\right)\|u\|_{X}^{2}+\|f\|_{2}^{2}\right] .
$$

Hence, by (4) and (5), it follows

(6) $\|\bar{w}\|_{X}^{2} \leq T_{1}\left\|\bar{u}_{0}\right\|_{H_{0}^{1}(\Omega)}^{2}+T_{1}^{2}\|w\|_{X}^{2} \leq$

$$
T_{1}\left\|\bar{u}_{0}\right\|_{H_{0}^{1}(\Omega)}^{2}+T_{1}^{2} \frac{\Lambda+1+c}{\lambda^{2}}\left[\left(\Lambda+T_{1}\|B\|_{L^{2}\left(0, T_{1} ; L^{\infty}(\Omega)\right)}^{2}\right)\|u\|_{X}^{2}+\|f\|_{2}^{2}\right] .
$$

Therefore, $H(X) \subset X$ and, taking into account (4), we have also $\bar{w}=H(u) \in H^{1}\left(\Omega \times\left(0, T_{1}\right)\right)$.

Next, let us prove that the operator $H$ is a contraction map. Indeed, given $u_{1}, u_{2} \in X$, we have that $\bar{w}:=H\left(u_{1}\right)-H\left(u_{2}\right)$ has null trace on the boundary $\partial \Omega, \bar{w}(x, 0)=0$ and it solves

$$
-\operatorname{div}(C(x, t) \nabla w)=\operatorname{div}\left(A(x, t) \nabla u+\int_{0}^{t} B(x, t-\tau) \nabla u(x, \tau) \mathrm{d} \tau\right),
$$

where $u=u_{1}-u_{2}$ and, according to (4), $w=\bar{w}_{t}$. Hence, by (6), we obtain

$$
\left\|H\left(u_{1}\right)-H\left(u_{2}\right)\right\|_{X}^{2}=\|\bar{w}\|_{X}^{2} \leq T_{1}^{2} \frac{\Lambda+1+c}{\lambda^{2}}\left(\Lambda+T_{1}\|B\|_{L^{2}\left(0, T_{1} ; L^{\infty}(\Omega)\right)}^{2}\right)\left\|u_{1}-u_{2}\right\|_{X}^{2} .
$$

Now, recalling that $T_{1} \leq T$, setting

$$
\gamma=\frac{\Lambda+1+c}{\lambda^{2}}\left(\Lambda+T\|B\|_{L^{2}\left(0, T_{1} ; L^{\infty}(\Omega)\right)}^{2}\right),
$$

and choosing $T_{1}=1 / 2 \sqrt{\gamma}$, we obtain that $H$ is a contraction. So, it admits a unique fixed point, i.e., a unique solution of (1) exists in $X$. Noting that the width $T_{1}$ of the time interval is independent of the iteration step, we may conclude the proof by iterating this argument over $(0, T)$.

Remark 2.1. Actually, (4) proves that the solution to problem (1) belongs to the space $H^{1}\left(0, T ; H_{0}^{1}(\Omega)\right)$.

\section{Main Result}

The aim of this section is to prove an existence and uniqueness result for the general non-coercive case. To this purpose, we first regularize the problem adding an $\varepsilon$-perturbation, $\varepsilon>0$, to the matrix $C$, in order to make it coercive. Then, we prove suitable uniform estimates with respect to $\varepsilon$. Finally, we obtain the desired result letting $\varepsilon \rightarrow 0$. Thus, for any $\varepsilon>0$, set

$$
C_{\varepsilon}(x, t)=C(x, t)+\varepsilon I,
$$

where $\varepsilon>0, I$ is the identity matrix and $C \in L^{\infty}\left(\Omega ; W^{1, \infty}\left(0, T ; \mathbb{R}^{N^{2}}\right)\right)$ satisfies

$$
\begin{aligned}
& C(x, t) \xi \cdot \xi \geq 0, \quad \text { for a.e. }(x, t) \in \Omega \times(0, T) \text { and every } \xi \in \mathbb{R}^{N}, \\
& C_{t}(x, t) \xi \cdot \xi \leq 0 \quad \text { for a.e. }(x, t) \in \Omega \times(0, T) \text { and every } \xi \in \mathbb{R}^{N} \text {, or }\left\|C_{t}\right\|_{\infty} \leq \lambda .
\end{aligned}
$$

For the sake of brevity, we employ the notation $\|D\|_{\infty}$ to denote the $L^{\infty}\left(\Omega \times(0, T) ; \mathbb{R}^{N^{2}}\right)$-norm of a given matrix $D \in L^{\infty}\left(\Omega \times(0, T) ; \mathbb{R}^{N^{2}}\right)$.

Notice that, when $C$ is independent of $t$, we are simply requiring that (9) holds and $C \in L^{\infty}\left(\Omega ; \mathbb{R}^{N^{2}}\right)$, while condition (10) is automatically satisfied. 
We assume also that $A, B \in L^{\infty}\left(\Omega \times(0, T) ; \mathbb{R}^{N^{2}}\right)$ and $A$ satisfies (2). For any $\varepsilon>0$, we consider the coercive problem

$$
\begin{array}{ll}
-\operatorname{div}\left(C_{\varepsilon} \nabla u_{t}^{\varepsilon}+A \nabla u^{\varepsilon}+\int_{0}^{t} B(x, t-\tau) \nabla u^{\varepsilon} \mathrm{d} \tau\right)=f, & \text { in } \Omega \times(0, T) ; \\
u^{\varepsilon}=0, & \text { on } \partial \Omega \times(0, T) ; \\
u^{\varepsilon}(x, 0)=\bar{u}_{0}(x), & \text { in } \Omega ;
\end{array}
$$

whose weak formulation is the following

$$
\begin{aligned}
\int_{0}^{T} \int_{\Omega} C_{\varepsilon} \nabla u_{t}^{\varepsilon} \cdot \nabla \varphi \mathrm{d} x \mathrm{~d} t & +\int_{0}^{T} \int_{\Omega} A \nabla u^{\varepsilon} \cdot \nabla \varphi \mathrm{d} x \mathrm{~d} t \\
& +\int_{0}^{T} \int_{\Omega}\left(\int_{0}^{t} B(x, t-\tau) \nabla u^{\varepsilon}(x, \tau) \mathrm{d} \tau\right) \cdot \nabla \varphi \mathrm{d} x \mathrm{~d} t=\int_{0}^{T} \int_{\Omega} f \varphi \mathrm{d} x \mathrm{~d} t,
\end{aligned}
$$

for all test functions $\varphi \in H^{1}(\Omega \times(0, T))$ such that $\left.\varphi\right|_{\partial \Omega}=0$ in $[0, T]$. By Theorem 2.1, it follows that, for any $\varepsilon>0$, there exists a unique solution $u^{\varepsilon} \in X$ of the previous problem, with the extra-regularity given in Remark 2.1.

Theorem 3.1. Let $A, B \in L^{\infty}\left(\Omega \times(0, T) ; \mathbb{R}^{N^{2}}\right)$ and $C \in L^{\infty}\left(\Omega ; W^{1, \infty}\left(0, T ; \mathbb{R}^{N^{2}}\right)\right)$. Assume that $A$ satisfies (2) and $C$ satisfies (9) and (10). Assume that $f \in L^{2}(\Omega \times(0, T)), \bar{u}_{0} \in H_{0}^{1}(\Omega)$ and let $u^{\varepsilon}$ be the solution of (11). Then, there exists $\gamma>0$, independent of $\varepsilon$, such that

$$
\left\|u^{\varepsilon}\right\|_{L^{2}\left(0, T ; H_{0}^{1}(\Omega)\right)} \leq \gamma .
$$

Proof. Choosing $\varphi=u^{\varepsilon}$ in (12) and taking into account (2), we obtain

$$
\begin{aligned}
\int_{0}^{T_{1}} \int_{\Omega} C_{\varepsilon} \nabla u_{t}^{\varepsilon} \cdot \nabla u^{\varepsilon} \mathrm{d} x & \mathrm{~d} t+\lambda \int_{0}^{T_{1}} \int_{\Omega}\left|\nabla u^{\varepsilon}\right|^{2} \mathrm{~d} x \mathrm{~d} t \\
\leq-\int_{0}^{T_{1}} \int_{\Omega} & \left(\int_{0}^{t} B \nabla u^{\varepsilon} \mathrm{d} \tau\right) \cdot \nabla u^{\varepsilon} \mathrm{d} x \mathrm{~d} t+\int_{0}^{T_{1}} \int_{\Omega} f u^{\varepsilon} \mathrm{d} x \mathrm{~d} t \\
& \leq \frac{\|B\|_{\infty}}{2} \int_{0}^{T_{1}} \int_{\Omega} \frac{d}{d t}\left(\int_{0}^{t}\left|\nabla u^{\varepsilon}(x, \tau)\right| \mathrm{d} \tau\right)^{2} \mathrm{~d} x \mathrm{~d} t+\frac{\|f\|_{2}^{2}}{2 \delta}+\frac{c \delta}{2}\left\|u^{\varepsilon}\right\|_{X},
\end{aligned}
$$

where $T_{1} \leq T$ will be chosen later, and $X=L^{2}\left(0, T_{1} ; H_{0}^{1}(\Omega)\right)$, as in the proof of Theorem 2.1. Then, integrating in time in the first integral on the left-hand side and on the right-hand side, it follows

$$
\begin{aligned}
\frac{1}{2} \int_{\Omega} C_{\varepsilon}\left(x, T_{1}\right) \nabla u^{\varepsilon}\left(x, T_{1}\right) \cdot \nabla u^{\varepsilon}\left(x, T_{1}\right) \mathrm{d} x+\lambda \int_{0}^{T_{1}} \int_{\Omega}\left|\nabla u^{\varepsilon}\right|^{2} \mathrm{~d} x \mathrm{~d} t \\
\leq \frac{1}{2} \int_{\Omega} C_{\varepsilon}(x, 0) \nabla \bar{u}_{0} \cdot \nabla \bar{u}_{0} \mathrm{~d} x+\frac{1}{2} \int_{0}^{T_{1}} \int_{\Omega} C_{t} \nabla u^{\varepsilon} \cdot \nabla u^{\varepsilon} \mathrm{d} x \mathrm{~d} t \\
+\frac{\|B\|_{\infty}}{2} \int_{\Omega}\left(\int_{0}^{T_{1}}|\nabla u(x, \tau)| \mathrm{d} \tau\right)^{2} \mathrm{~d} x+\frac{\|f\|_{2}^{2}}{2 \delta}+\frac{c \delta}{2}\left\|u^{\varepsilon}\right\|_{X}^{2} \\
\leq \frac{1}{2} \int_{\Omega} C_{\varepsilon}(x, 0) \nabla \bar{u}_{0} \cdot \nabla \bar{u}_{0} \mathrm{~d} x+\frac{1}{2} \int_{0}^{T_{1}} \int_{\Omega} C_{t} \nabla u^{\varepsilon} \cdot \nabla u^{\varepsilon} \mathrm{d} x \mathrm{~d} t \\
\quad+\frac{T_{1}\|B\|_{\infty}}{2}\left\|u^{\varepsilon}\right\|_{X}^{2}+\frac{\|f\|_{2}^{2}}{2 \delta}+\frac{c \delta}{2}\left\|u^{\varepsilon}\right\|_{X}^{2},
\end{aligned}
$$

where we recall that (8) implies $C_{\varepsilon t}=C_{t}$. Now, taking into account (10), choosing $\delta=\frac{\lambda}{2 c}$ and $T_{1}=\frac{\lambda}{4\|B\|_{\infty}}$, 
we can absorb the second, the third and the fifth term in the left-hand side, thus getting

$$
\begin{aligned}
\int_{0}^{T_{1}} \int_{\Omega}\left|\nabla u^{\varepsilon}\right|^{2} \mathrm{~d} x \mathrm{~d} t & \leq \frac{4}{\lambda} \int_{\Omega} C_{\varepsilon}(x, 0) \nabla \bar{u}_{0} \cdot \nabla \bar{u}_{0} \mathrm{~d} x+\frac{8\|f\|_{2}^{2}}{\lambda^{2}} \\
& \leq \frac{4}{\lambda}\left(\|C\|_{\infty}+1\right)\left\|\bar{u}_{0}\right\|_{H_{0}^{1}(\Omega)}^{2}+\frac{8\|f\|_{2}^{2}}{\lambda^{2}} \\
\int_{\Omega} C_{\varepsilon}\left(x, T_{1}\right) \nabla u^{\varepsilon}\left(x, T_{1}\right) \cdot \nabla u^{\varepsilon}\left(x, T_{1}\right) \mathrm{d} x & \leq \int_{\Omega} C_{\varepsilon}(x, 0) \nabla \bar{u}_{0} \cdot \nabla \bar{u}_{0} \mathrm{~d} x+\frac{2\|f\|_{2}^{2}}{\lambda} \\
& \leq\left(\|C\|_{\infty}+1\right)\left\|\bar{u}_{0}\right\|_{H_{0}^{1}(\Omega)}^{2}+\frac{2\|f\|_{2}^{2}}{\lambda} .
\end{aligned}
$$

Repeating the same argument in $\left(T_{1}, 2 T_{1}\right)$, by (14) and (15), we obtain

$$
\begin{aligned}
\int_{T_{1}}^{2 T_{1}} \int_{\Omega}\left|\nabla u^{\varepsilon}\right|^{2} \mathrm{~d} x \mathrm{~d} t \leq \frac{4}{\lambda} \int_{\Omega} C_{\varepsilon}\left(x, T_{1}\right) \nabla u^{\varepsilon}\left(x, T_{1}\right) \cdot & \nabla u^{\varepsilon}\left(x, T_{1}\right) \mathrm{d} x+\frac{8\|f\|_{2}^{2}}{\lambda^{2}} \\
& \leq \frac{4}{\lambda}\left(\left(\|C\|_{\infty}+1\right)\left\|\bar{u}_{0}\right\|_{H_{0}^{1}(\Omega)}^{2}+\frac{2\|f\|_{2}^{2}}{\lambda}\right)+\frac{8\|f\|_{2}^{2}}{\lambda^{2}},
\end{aligned}
$$

and

$$
\begin{aligned}
\int_{\Omega} C_{\varepsilon}\left(x, 2 T_{1}\right) \nabla u^{\varepsilon}\left(x, 2 T_{1}\right) & \cdot \nabla u^{\varepsilon}\left(x, 2 T_{1}\right) \mathrm{d} x \\
\leq \int_{\Omega} C_{\varepsilon}\left(x, T_{1}\right) \nabla u^{\varepsilon}\left(x, T_{1}\right) \cdot \nabla u^{\varepsilon}\left(x, T_{1}\right) \mathrm{d} x+\frac{2\|f\|_{2}^{2}}{\lambda} & \leq\left(\|C\|_{\infty}+1\right)\left\|\bar{u}_{0}\right\|_{H_{0}^{1}(\Omega)}^{2}+\frac{2\|f\|_{2}^{2}}{\lambda}+\frac{2\|f\|_{2}^{2}}{\lambda} .
\end{aligned}
$$

Hence, since only a finite number $n$ of steps is required, in order to recover the whole interval $(0, T)$, it follows that

$$
\left\|u^{\varepsilon}\right\|_{L^{2}\left(0, T ; H_{0}^{1}(\Omega)\right)}^{2}=\sum_{i=1}^{n} \int_{T_{i-1}}^{T_{i}} \int_{\Omega}\left|\nabla u^{\varepsilon}\right|^{2} \mathrm{~d} x \mathrm{~d} t+\int_{T_{n}}^{T} \int_{\Omega}\left|\nabla u^{\varepsilon}\right|^{2} \mathrm{~d} x \mathrm{~d} t \leq \gamma,
$$

where we set $T_{0}=0$ and the bound $\gamma$ does not depend on $\varepsilon$. This concludes the proof.

As a consequence of Theorem 3.1, there exists a function $u \in L^{2}\left(0, T ; H_{0}^{1}(\Omega)\right)$ such that, up to a subsequence,

$$
\begin{array}{ll}
u^{\varepsilon} \rightarrow u & \text { weakly in } L^{2}(\Omega \times(0, T)), \\
\nabla u^{\varepsilon} \rightarrow \nabla u & \text { weakly in } L^{2}(\Omega \times(0, T)) .
\end{array}
$$

Theorem 3.2. Let $A, B \in L^{\infty}\left(\Omega \times(0, T) ; \mathbb{R}^{N^{2}}\right)$ and $C \in L^{\infty}\left(\Omega ; W^{1, \infty}\left(0, T ; \mathbb{R}^{N^{2}}\right)\right)$. Assume that $A$ satisfies (2) and $C$ satisfies (9) and (10). Assume that $f \in L^{2}(\Omega \times(0, T))$ and $\bar{u}_{0} \in H_{0}^{1}(\Omega)$. Then, there exists a unique function $u \in L^{2}\left(0, T ; H_{0}^{1}(\Omega)\right)$ satisfying problem (1) in the sense (18) below, for all test functions $\varphi \in H^{1}\left(0, T ; H_{0}^{1}(\Omega)\right)$ such that $\varphi(x, T)=0$ a.e. in $\Omega$.

Proof. Integrating by parts with respect to the time $t$ the first integral in (12), recalling that $C_{\varepsilon t}=C_{t}$, and passing to the limit for $\varepsilon \rightarrow 0$, by (16) and (17), it follows

$$
\begin{aligned}
-\int_{0}^{T} \int_{\Omega} C \nabla u \cdot \nabla \varphi_{t} \mathrm{~d} x & \mathrm{~d} t-\int_{0}^{T} \int_{\Omega} C_{t} \nabla u \cdot \nabla \varphi \mathrm{d} x \mathrm{~d} t+\int_{0}^{T} \int_{\Omega} A \nabla u \cdot \nabla \varphi \mathrm{d} x \mathrm{~d} t \\
+\int_{0}^{T} \int_{\Omega}\left(\int_{0}^{t} B(x, t-\tau) \nabla u(x, \tau) \mathrm{d} \tau\right) \cdot \nabla \varphi \mathrm{d} x \mathrm{~d} t & =\int_{0}^{T} \int_{\Omega} f \varphi \mathrm{d} x \mathrm{~d} t+\int_{\Omega} C(x, 0) \nabla \bar{u}_{0} \cdot \nabla \varphi(x, 0) \mathrm{d} x
\end{aligned}
$$


for all test functions $\varphi \in H^{1}\left(0, T ; H_{0}^{1}(\Omega)\right)$ such that $\varphi(x, T)=0$ a.e. in $\Omega$. Here, we used the fact that, by (8), $C_{\varepsilon} \rightarrow C$ strongly in $L^{2}\left(\Omega \times(0, T) ; \mathbb{R}^{N^{2}}\right)$. Moreover, setting

$$
g_{\varepsilon}(t)=\int_{0}^{t} \int_{\Omega} B(x, t-\tau) \nabla u^{\varepsilon}(x, \tau) \mathrm{d} x \mathrm{~d} \tau \quad \text { and } \quad g(t)=\int_{0}^{t} \int_{\Omega} B(x, t-\tau) \nabla u(x, \tau) \mathrm{d} x \mathrm{~d} \tau,
$$

we have that $g_{\varepsilon}(t) \rightarrow g(t)$ pointwise a.e. in $(0, T)$ and, by Theorem $3.1,|g(t)| \leq \gamma\|B\|_{\infty}\left\|\nabla u^{\varepsilon}\right\|_{2}^{2} \leq \gamma$, so that $g_{\varepsilon} \rightarrow g$ strongly in $L^{2}(0, T)$. Therefore, $u$ is a weak solution of problem (1). In order to prove the uniqueness, we proceed as follows. Set $U=u_{1}-u_{2}$, where $u_{i}, i=1,2$ are two different solutions of (1). Then, $U \in L^{2}\left(0, T ; H_{0}^{1}(\Omega)\right)$ satisfies (1) with $f \equiv 0$ and homogeneous boundary and initial conditions. Moreover, by the energy estimate, we get

$$
\begin{array}{rl}
0=\int_{0}^{T_{1}} \int_{\Omega} C \nabla U_{t} \cdot \nabla U \mathrm{~d} & \mathrm{~d} t \\
+\int_{0}^{T_{1}} \int_{\Omega} A \nabla U \cdot \nabla U \mathrm{~d} x \mathrm{~d} t \\
+\int_{0}^{T_{1}} \int_{\Omega}\left(\int_{0}^{t} B(x, t-\tau) \nabla U(x, \tau) \mathrm{d} \tau\right) \cdot \nabla U \mathrm{~d} x \mathrm{~d} t \\
=\frac{1}{2} \int_{\Omega} C\left(x, T_{1}\right) \nabla U\left(x ; T_{1}\right) \cdot \nabla U\left(x, T_{1}\right) \mathrm{d} x-\frac{1}{2} \int_{0}^{T_{1}} \int_{\Omega} C_{t} \nabla U \cdot \nabla U \mathrm{~d} x \mathrm{~d} t+\int_{0}^{T_{1}} \int_{\Omega} A \nabla U \cdot \nabla U \mathrm{~d} x \mathrm{~d} t \\
+\int_{0}^{T_{1}} \int_{\Omega}\left(\int_{0}^{t} B(x, t-\tau) \nabla U(x, \tau) \mathrm{d} \tau\right) \cdot \nabla U \mathrm{~d} x \mathrm{~d} t,
\end{array}
$$

and therefore, reasoning as in (13), it follows

$$
\lambda \int_{0}^{T_{1}} \int_{\Omega}|\nabla U|^{2} \mathrm{~d} x \mathrm{~d} t \leq\left(\frac{\lambda+T_{1}\|B\|_{\infty}}{2}\right) \int_{0}^{T_{1}} \int_{\Omega}|\nabla U|^{2} \mathrm{~d} x \mathrm{~d} t .
$$

If we choose $T_{1}=\frac{\lambda}{4\|B\|_{\infty}}$, this implies that $\int_{0}^{T_{1}} \int_{\Omega}|\nabla U|^{2} \mathrm{~d} x \mathrm{~d} t \leq 0$, so that $u_{1}=u_{2}$ a.e. in $\Omega \times\left(0, T_{1}\right)$. Since $T_{1}$ depends only on $\lambda$ and $\|B\|_{\infty}$, we repeat the same argument for a finite number of steps and we get that $u_{1}=u_{2}$ in the whole $\Omega \times(0, T)$. This, in particular, implies that the whole sequence $u^{\varepsilon} \rightarrow u$. This concludes the proof.

Remark 3.1. We may consider a non homogeneous boundary condition $u=g$ on $\partial \Omega \times(0, T)$. To this purpose, it is enough to assume $g \in H^{1}\left(0, T ; H^{1}(\Omega)\right)$ with $g(\cdot, 0) \in H_{0}^{1}(\Omega), B \in L^{\infty}\left(\Omega \times(0, T) ; \mathbb{R}^{N^{2}}\right)$ and to replace in (1) $u$ with $v=u-g, \bar{u}_{0}$ with $u_{0}=\bar{u}_{0}-g(x, 0)$ and $f$ with

$$
\tilde{f}(x, t)=f(x, t)-\operatorname{div}\left(C(x, t) \nabla g_{t}+A(x, t) \nabla g+\int_{0}^{t} B(x, t-\tau) \nabla g(x, \tau) \mathrm{d} \tau\right) .
$$

It is worthwhile to notice that all the proofs can be carried out in essentially the same way for $\tilde{f} \in$ $L^{2}\left(0, T ; H^{-1}(\Omega)\right)$.

\section{Acknowledgements}

The first author is member of the Gruppo Nazionale per l'Analisi Matematica, la Probabilità e le loro Applicazioni (GNAMPA) of the Istituto Nazionale di Alta Matematica (INdAM). The second author is member of the Gruppo Nazionale per la Fisica Matematica (GNFM) of the Istituto Nazionale di Alta Matematica (INdAM). The last author wishes to thank Dipartimento di Scienze di Base e Applicate per l'Ingegneria for the warm hospitality and Università "La Sapienza" of Rome for the financial support.

\section{References}

1. M. Amar, D. Andreucci, P. Bisegna, and R. Gianni, Stability and memory effects in a homogenized model governing the electrical conduction in biological tissues, Journal of Mechanics of Materials and Structures, vol. 4, pp. 211-223, 2009. 
2. M. Amar, D. Andreucci, P. Bisegna, and R. Gianni, Homogenization limit and asymptotic decay for electrical conduction in biological tissues in the high radiofrequency range, Communications on Pure and Applied Analysis, vol. 9, pp. 1131-1160, 2010.

3. M. Amar, D. Andreucci, R. Gianni, and C. Timofte, Concentration and homogenization in electrical conduction in heterogeneous media involving the Laplace-Beltrami operator, Submitted (2018).

4. M. Amar, D. Andreucci, P. Bisegna, and R. Gianni, Homogenization limit for electrical conduction in biological tissues in the radio-frequency range, Comptes Rendus Mécanique, vol. 331, pp. 503-508, 2003. Elsevier.

5. M. Amar, D. Andreucci, P. Bisegna, and R. Gianni, Evolution and memory effects in the homogenization limit for electrical conduction in biological tissues, Mathematical Models and Methods in Applied Sciences, vol. 14, pp. 1261-1295, 2004. World Scientific.

6. M. Amar, D. Andreucci, P. Bisegna, and R. Gianni, On a hierarchy of models for electrical conduction in biological tissues, Mathematical Methods in the Applied Sciences, vol. 29, pp. 767-787, 2006.

7. M. Amar, D. Andreucci, P. Bisegna, and R. Gianni, Exponential asymptotic stability for an elliptic equation with memory arising in electrical conduction in biological tissues, European Journal of Applied Mathematics, vol. 20, pp. 431-459, 2009.

8. M. Amar, D. Andreucci, P. Bisegna, and R. Gianni, An elliptic equation with history, C. R. Acad. Sci. Paris, Ser. I, vol. 338, pp. 595-598, 2004. Elsevier.

9. G. Barenblatt, V. Entov, and V. Ryzhik, Theory of Fluid Flow Through Natural Rocks. Kluwer, Dordrecht, 1990.

10. M. G. Crandall, S.-O. Londen, and J. A. Nohel, An abstract nonlinear Volterra integrodifferential equation, Journal of Mathematical Analysis and Applications, vol. 64, no. 3, pp. 701-735, 1978.

11. M. Fabrizio, Sul problema di Fichera in viscoelasticità lineare, in Atti del IV Meeting: Waves and Stability, Bari, 1989.

12. G. Fichera, Integro-differential problems of hereditary elasticity, Atti del Seminario Matematico $e$ Fisico dell'Università di Modena, vol. 26, no. 2, pp. 363-370 (1979), 1977.

13. C. Giorgi and B. Lazzari, On the stability for linear viscoelastic solids, Quarterly of Applied Mathematics, vol. 55, no. 4, pp. 659-675, 1997.

14. W. Gray and S. Hassanizadeh, Thermodynamic basis of capillary pressure in porous media, Water Resources Research, vol. 29, pp. 3389-3405, 1993.

15. M. L. Heard, An abstract parabolic Volterra integro-differential equation, SIAM Journal on Mathematical Analysis, vol. 13, no. 1, pp. 81-105, 1982.

16. R. K. Miller, An integro-differential equation for rigid heat conductors with memory, Journal of Mathematical Analysis and Applications, vol. 66, no. 2, pp. 313-332, 1978.

17. M. Ptashnyk, Degenerate quaslinear pseudoparabolic equations with memory terms and variational inequalities, Nonlinear Analysis, vol. 66, no. 12, pp. 2653-2675, 2007.

18. L. Tartar, Memory effects and homogenization, Archive for Rational Mechanics and Analysis, vol. 111, no. 2, pp. 121-133, 1990. 